OPEN ACCESS

Edited by:

Andrea Martinuzzi,

Eugenio Medea (IRCCS), Italy

Reviewed by:

Andrzej Szopa,

Medical University of Silesia, Poland

Giulia Stamjpacchia,

Pisana University Hospital, Italy

${ }^{*}$ Correspondence:

Faustyna Manikowska foka@interia.p

Specialty section:

This article was submitted to

Neurorehabilitation,

a section of the journa

Frontiers in Neurology

Received: 11 June 2021 Accepted: 11 November 2021 Published: 23 December 2021

Citation:

Manikowska F, Brazevic S, Krzyżańska A and Jóźwiak M (2021) Effects of Robot-Assisted Therapy on Gait Parameters in Pediatric Patients

With Spastic Cerebral Palsy.

Front. Neurol. 12:724009. doi: 10.3389/fneur.2021.724009

\section{Effects of Robot-Assisted Therapy on Gait Parameters in Pediatric Patients With Spastic Cerebral Palsy}

\author{
Faustyna Manikowska ${ }^{1 *}$, Sabina Brazevic ${ }^{1}$, Anna Krzyżańska ${ }^{2}$ and Marek Jóźwiak ${ }^{1}$ \\ ${ }^{1}$ Gait and Motion Analysis Laboratory, Poznań University of Medical Sciences, Poznań, Poland, ${ }^{2}$ X-Rehab, Poznań \\ Rehabilitation Center, Poznań, Poland
}

Background: Gait dysfunction is a crucial factor that restricts independence and quality of life in children with cerebral palsy (CP). Gait training based on robotic-assisted therapy (RAT) is widely used, but information about effectiveness and ideal patient profile is not sufficient. Aim of this study was to assess the effect of RAT on gait parameters in spastic children with $\mathrm{CP}$, and to determine whether changes in gait parameters are different among patients on different ambulatory levels.

Method: A total of 26 children with bilateral spastic CP were divided into two groups based on their functional ability: non-assisted ambulator (NAS) or assisted ambulator (AS); and underwent a RAT program (30 training sessions of RAT during 10 weeks). Gait analysis was performed: before the therapy (t1), right after (t2), and 6 weeks later (t3).

Results: No significant changes in spatiotemporal parameters or gait deviation index at $\mathrm{t} 2$ or $\mathrm{t} 3$. Double support symmetry significantly improved (t1 vs. $\mathrm{t} 3, p=0.03$ ) for the whole group (NAS + AS). Walking speed symmetry significantly improved (t2 vs. t3, $p=0.02)$ for group AS.

Conclusion: RAT based on our protocol did not change spatiotemporal parameters and kinematics of walking except limited improvement in some aspects of gait symmetry. We did not find differences in changes in selected objective gait parameters among children with $\mathrm{CP}$ in different ambulatory levels.

Keywords: gait analysis, balance, symmetry index, gait deviation index, spatiotemporal parameters

\section{INTRODUCTION}

Cerebral palsy (CP) is a disorder with a non-homogeneous combination of symptoms that leads to disability. In most cases, the mixture of positive (increased muscle activities) and negative (insufficient active muscle control) signs of motor disorders can be presented $(1,2)$. Various combinations of simultaneous present and severity of impairments are observed as the clinical conditions of an individual subject and make an impact on their functional status (3). Because of that, therapy for this population should be individualized and task-oriented for each patient (4).

Gait dysfunction is a crucial factor that restricts independence and quality of life (5-7). Meta-analysis has shown that gait training is the most effective intervention to improve gait parameters in patients with $\mathrm{CP}(8)$. The robot-assisted therapy (RAT) has been combined to the 
conventional gait training recently (9). It has been developed and improved to meet the needs of each patient. Based on the real-time feedback and collected data, individual differences on therapy, including time, endurance, and level of advance can be adapted $(10,11)$. It was suggested that robot-assisted gait training is an effective therapy for this population $(8,12-14)$.

Despite the fact that the new generation of RAT can meet individual requirements better than conventional training in theory and may lead to better functional outcomes, the amount and quality of research in this field is still very limited (8). Training protocols mainly focused on gait training and outcomes measures of the therapy differ from study to study (12-15). Most of the studies focused on walking endurance, walking speed, and functional assessment of walking ability with varying numbers of therapy sessions from 1 to 20 (16, 17). Moreover, small sample size is a very common limitation $(14,18-20)$.

The primary aim of the study was to assess the effect of RAT on selective objective gait parameters in children with spastic $\mathrm{CP}$. The secondary aim was to determine whether changes in selected objective gait parameters are different among children in different ambulatory levels. To achieve the aforementioned aims, we investigated the short-term changes in gait kinematic data, gait symmetry, and gait spatiotemporal parameters from gait analyses performed before and after RAT, in the two functional groups: independent ambulators and dependent ambulators who use assistive devices.

\section{MATERIALS AND METHODS}

\section{Participants}

Children were recruited to participate in this prospective study. Inclusion criteria were: (1) diagnosis of bilateral spastic CP, (2) able to follow verbal instructions, (3) gait training was the aim of the therapy, (4) body height at least $150 \mathrm{~cm}$, and (5) only received conventional therapy within the past 6 months. Exclusion criteria were: (1) received surgery or botulinum toxin injection within the past 6 months, (2) pain or fixed contractures in the lower limb joints that prevent from applying RAT.

In total, 26 children with bilateral spastic $\mathrm{CP}$ were recruited to the study (female, $n=10$; male, $n=16$; age $=14.8$ [1.97] years; range: $12-18$ years). All the participants underwent a RAT program in the outpatient service of a local rehabilitation hospital and were examined in the motion analysis laboratory of the university hospital. Participants were divided into two groups based on their baseline functional ability, that is, Gross Motor Function Classification System (GMFCS) level, before the therapy program started: Group NAS (non-assisted ambulator, $n=17$ ) were those who could walk independently (GMFCS levels I and II), and Group AS (assisted ambulator, $n=9$ ) were those who walk with assistive devices (GMFCS levels III \& IV).

The study received appropriate approval from the Institutional Review Board of the Poznan University of Medical Sciences ( $\mathrm{nr}$ 681/18, June 16, 2018). Written consents were acquired from all the participants. For those younger than 18 years, consents were obtained from the legal guardian.

\section{Protocol}

Anthropometric data (age, body weight, and body height) were collected from all the participants at their first visit.

\section{Robot-Assisted Therapy Program (Gait Training) Protocol}

Each patient received a 10 -week (30 sessions) RAT protocol in the following order: training (2 weeks; five sessions per week), break ( 2 weeks), training (2 weeks; five sessions per week), break (2 weeks), training (2 weeks; five sessions per week). This therapy protocol was created based on the Camp's formula in order to assess the effectiveness of an intensive, complex, and long-term RAT, and to monitor physical activities and the physical therapy during the time the present study was conducted. The 2-week break allows participants to return their residential place temporarily because of emotional, family, and employment needs.

During the first session, the participants got familiar with the therapy program. On the 1st day, the therapy dose was optimized to meet individual capability of each patient. At each equipment, the diagnostic tests were done. Balance, ability to shift the center of pressure, spatiotemporal gait parameters, endurance, and the level of required support during walking were assessed. Based on these data, the baseline level for each patient was established and it was a starting point for individual therapy. During the RAT based on the real-time assessment (based on the same diagnostic tests) the progress was detected and the training difficulty was adjusted. Virtual reality games were integrated into treadmill and platforms. The amount of support for the exoskeleton system was established on the first session. Based on the feedback information from each lower limb joint, the support level for each leg was established and adjusted.

The functional gait training was based on RAT. Because the dynamic balance influences walking pattern, we decided that each session consists of: gait training (with exoskeleton and treadmill) and balance training (on stabilometric and dynamographic platforms) according to the therapy protocol:

\section{- 10 min on Gamma VAST (AC International East):}

analysis of load distribution between left and right sides of the body side (for balance training)

create individual training difficulty by the real-time biofeedback

- 5-min break

- 10 min on Alfa VAST (AC International East):

- dynamic analysis of center of pressure displacement

- training of balance according to amount of displacement

- 10-min break

- 45 min of EksoGT (Ekso Bionics):

- analysis of required support of each lower limb joint

- customized gait training with different level of support.

The training starts from a shorter period of time (10-15 min). Depends on the endurance, the usual walking time range from $30 \mathrm{~min}$ to $1 \mathrm{~h}$. 
- 15-min break

- $2 \times 15$ min on zebris THQ-M-3i Treadmill (zebris Medical $\mathrm{GmbH})$

- analysis of spatiotemporal gait parameters and endurance virtual reality trainings for walking balance and gait

- 5-min break

The whole therapy program was performed under the supervision of a physical therapist experienced with RAT.

\section{Gait Analysis}

Gait analysis was performed at the following three time points: before the therapy program started ( $\mathrm{t} 1)$, right after all 30 training sessions were completed ( $\mathrm{t} 2$ ), and 6 weeks after the therapy program was done $(\mathrm{t} 3)$.

Kinematic data were collected with an eight-camera threedimensional gait analysis system (six Bonita cameras and two Vero cameras; Vicon Motion Systems Ltd., Oxford, United Kingdom) sampling at $100 \mathrm{~Hz}$. Reflective markers were applied according to the standard Plug-in-Gait marker placement model to each patient. Participants walk barefoot along a 10-m walkway with self-selected speed.

\section{Data Analysis}

\section{Statistical Analysis}

The normality of the distribution of variables was tested with the Shapiro-Wilk test. To investigate the changes over time of the analyzed parameters, in case of compliance with the normal distribution and the homogeneity of variance, repeatedmeasures ANOVA with Tukey's multiple comparisons test was calculated. In the remaining cases, the Friedman test with the Dunn-Bonferroni multiple comparison test was calculated. In order to assess whether the changes in the parameters analyzed had deterioration, no change, improvement over time are statistically significant, the McNemar-Bowker symmetry test was used. To compare the data between ambulators and nonambulators, $t$-test, Cochran-Cox test, or Mann-Whitney test was used depending upon the distribution of normality and variance equality. A power analysis was performed to estimate the appropriate sample size. All the calculations were made using Statistical version 12 (TIBCO Software Inc., Palo Alto, California, USA). Statistical significance was set at the $p$-value of 0.05 .

\section{Outcome Measure}

Primary outcome measures were: spatiotemporal gait parameters, including walking speed $(\mathrm{m} / \mathrm{s})$, cadence (steps/min), step time (s), step length $(\mathrm{m})$, stride time (s), stride length $(\mathrm{m})$, step width $(\mathrm{m})$, foot off ( $\%$ of the gait cycle), double support $(\%$ of the gait cycle), single support (\% of the gait cycle), opposite foot off ( $\%$ of the gait cycle), and opposite foot contact ( $\%$ of the gait cycle). All spatiotemporal parameters were processed in the default data collection software provided by the instrumental gait analysis system manufacturer (Vicon Motion Systems Ltd., Oxford, UK) and extracted directly from the standard C3D format file from a custom-coded program under MATLAB (MathWorks, Inc., Natick, MA, USA) environment. Gait symmetry of all parameters were calculated by Symmetry Index (SI) and Gait Deviation Index (GDI) $(21,22)$.

Symmetry Index was calculated according to the formula (23):

$$
S I=\frac{2\left(X_{R}-X_{L}\right)}{X_{R}+X_{L}} \times 100 \%
$$

$\mathrm{X}_{\mathrm{R}}$ : right leg parameter

$\mathrm{X}_{\mathrm{L}}$ : left leg parameter

* Signs before the SI value indicate the direction of asymmetry: negative means to the left, positive to the right. SI $=0$ indicates full symmetry.

Changes in the gait pattern of each subject were categorized into one of the three following groups according to the change of GDI between visits $(23,24)$ : improvement $(\Delta \mathrm{GDI} \geq 5)$, no change $(-5<\Delta \mathrm{GDI}<5)$, or deterioration $(\Delta \mathrm{GDI} \leq-5)$.

\section{RESULTS}

\section{Spatiotemporal Parameters}

After receiving RAT, we did not find statistically significant changes in spatiotemporal parameters neither right after therapy (t2) nor 6 weeks after therapy ( $\mathrm{t} 3$ ). The same analysis was done in functional subgroups and no statistically significant changes were found either (Table 1).

\section{Gait Symmetry}

Analysis of SI for spatiotemporal parameters showed significant changes in double support symmetry while considering all participants as one group, i.e., NAS + AS $(\mathrm{t} 2=0.04, \mathrm{t} 3=-0.01$; $p=0.03$; Figure 1A), and walking speed symmetry for patient walking with aids, i.e., AS ( $\mathrm{t} 1=-0.04$, $\mathrm{t} 3=0.02 ; p=0.02$; Figure 1B) (Table 2).

\section{Gait Deviation Index}

Taking all the 26 participants as one group, the GDI at baseline (t1) was: mean $(\mathrm{SD})=73.36(11.28), \min =55.19$, $\max =100.52$, median $=71.56$. We did not find any significant change in GDI neither right after therapy ( $\mathrm{t} 2$ : mean $[\mathrm{SD}]=72.62$ [12.20], $\min =53.45, \max =102.39$, median $=72.17$ ) nor 6 weeks after the last session ( $\mathrm{t} 3$ : mean $[\mathrm{SD}]=74.02[13.29], \mathrm{min}=56.63$, $\max =102.71$, median $=71.11)(p=0.45)$.

While focused on the change of GDI (improvement, no change, or deterioration), the analysis showed that changes right after therapy (t2) and after 6 weeks (t3) were not statistically significant (Table 3). The same analysis was performed based on functional subgroups. No significant change was found in either Group NAS $(p=0.80)$ or Group AS $(p=0.92)$.

Analysis of GDI symmetry between left and right sides did not show statistically significant changes between visits neither for the whole study group (NAS $+\mathrm{AS}, p=0.31$ ), nor for subgroups (NAS, $p=0.40$; AS, $p=0.36$ ).

Comparing differences between the two groups, the statistical power was in the range $32.3-74.2 \%$. Comparing the differences in time, the statistical power was in the range of $91.0-92.0 \%$. 
TABLE 1 | Changes in spatiotemporal parameters between visits.

\begin{tabular}{|c|c|c|c|c|c|c|c|c|c|c|c|c|}
\hline \multirow[t]{3}{*}{ Parameter } & \multicolumn{4}{|c|}{ NAS + AS $(n=26)$} & \multicolumn{4}{|c|}{ NAS $(n=17)$} & \multicolumn{4}{|c|}{ AS $(n=9)$} \\
\hline & $\begin{array}{c}\text { Mean } \\
\text { (SD) }\end{array}$ & $\begin{array}{l}\text { Mean } \\
\text { (SD) }\end{array}$ & $\begin{array}{c}\text { Mean } \\
\text { (SD) }\end{array}$ & $p$ & $\begin{array}{c}\text { Mean } \\
\text { (SD) }\end{array}$ & $\begin{array}{c}\text { Mean } \\
\text { (SD) }\end{array}$ & $\begin{array}{c}\text { Mean } \\
\text { (SD) }\end{array}$ & $p$ & $\begin{array}{c}\text { Mean } \\
\text { (SD) }\end{array}$ & $\begin{array}{c}\text { Mean } \\
\text { (SD) }\end{array}$ & $\begin{array}{c}\text { Mean } \\
\text { (SD) }\end{array}$ & $p$ \\
\hline & t1 & t2 & t3 & & $t 1$ & t2 & t3 & & t1 & t2 & t3 & \\
\hline Cadence (step/min) & $\begin{array}{c}84.32 \\
(25.87)\end{array}$ & $\begin{array}{l}85.36 \\
(24.0)\end{array}$ & $\begin{array}{c}85.80 \\
(23.23)\end{array}$ & 0.41 & $\begin{array}{c}94.71 \\
(16.73)\end{array}$ & $\begin{array}{c}95.06 \\
(12.41)\end{array}$ & $\begin{array}{l}97.80 \\
(9.05)\end{array}$ & 0.79 & $\begin{array}{c}64.70 \\
(29.49)\end{array}$ & $\begin{array}{c}67.04 \\
(30.23)\end{array}$ & $\begin{array}{c}63.13 \\
(25.28)\end{array}$ & 0.74 \\
\hline Double support (s) & $\begin{array}{c}0.73 \\
(0.99)\end{array}$ & $\begin{array}{c}0.66 \\
(0.79)\end{array}$ & $\begin{array}{c}0.65 \\
(0.77)\end{array}$ & 0.41 & $\begin{array}{c}0.43 \\
(0.29)\end{array}$ & $\begin{array}{c}0.40 \\
(0.19)\end{array}$ & $\begin{array}{c}0.34 \\
(0.11)\end{array}$ & 0.26 & $\begin{array}{c}1.30 \\
(1.53)\end{array}$ & $\begin{array}{c}1.16 \\
(1.21)\end{array}$ & $\begin{array}{c}1.23 \\
(1.11)\end{array}$ & 0.46 \\
\hline Foot off (\%) & $\begin{array}{l}67.29 \\
(8.85)\end{array}$ & $\begin{array}{l}67.01 \\
(9.38)\end{array}$ & $\begin{array}{l}66.87 \\
(8.32)\end{array}$ & 0.15 & $\begin{array}{l}65.02 \\
(5.14)\end{array}$ & $\begin{array}{l}64.91 \\
(4.79)\end{array}$ & $\begin{array}{l}63.46 \\
(3.34)\end{array}$ & 0.08 & $\begin{array}{c}71.57 \\
(12.66)\end{array}$ & $\begin{array}{c}70.97 \\
(14.22)\end{array}$ & $\begin{array}{c}73.32 \\
(11.06)\end{array}$ & 0.60 \\
\hline Opposite foot contact (\%) & $\begin{array}{l}49.94 \\
(0.76)\end{array}$ & $\begin{array}{l}50.22 \\
(0.88)\end{array}$ & $\begin{array}{l}50.11 \\
(0.65)\end{array}$ & 0.40 & $\begin{array}{l}49.85 \\
(0.73)\end{array}$ & $\begin{array}{l}50.02 \\
(0.69)\end{array}$ & $\begin{array}{l}50.01 \\
(0.63)\end{array}$ & 0.69 & $\begin{array}{l}50.11 \\
(0.84)\end{array}$ & $\begin{array}{l}50.58 \\
(1.11)\end{array}$ & $\begin{array}{l}50.31 \\
(0.67)\end{array}$ & 0.89 \\
\hline Opposite foot off (\%) & $\begin{array}{l}17.38 \\
(9.24)\end{array}$ & $\begin{array}{l}17.30 \\
(9.57)\end{array}$ & $\begin{array}{l}17.01 \\
(8.22)\end{array}$ & 0.45 & $\begin{array}{l}15.10 \\
(5.36)\end{array}$ & $\begin{array}{l}14.97 \\
(4.39)\end{array}$ & $\begin{array}{l}13.66 \\
(3.40)\end{array}$ & 0.33 & $\begin{array}{c}21.67 \\
(13.32)\end{array}$ & $\begin{array}{c}21.69 \\
(14.64)\end{array}$ & $\begin{array}{c}23.35 \\
(10.91)\end{array}$ & 0.72 \\
\hline Single support (s) & $\begin{array}{c}0.48 \\
(0.14)\end{array}$ & $\begin{array}{c}0.47 \\
(0.12)\end{array}$ & $\begin{array}{c}0.47 \\
(0.08)\end{array}$ & 0.96 & $\begin{array}{c}0.45 \\
(0.06)\end{array}$ & $\begin{array}{c}0.45 \\
(0.05)\end{array}$ & $\begin{array}{c}0.45 \\
(0.04)\end{array}$ & 0.90 & $\begin{array}{c}0.54 \\
(0.23)\end{array}$ & $\begin{array}{c}0.52 \\
(0.19)\end{array}$ & $\begin{array}{l}0.51 \\
(0.11)\end{array}$ & 0.97 \\
\hline Step length (m) & $\begin{array}{c}0.45 \\
(0.14)\end{array}$ & $\begin{array}{c}0.44 \\
(0.15)\end{array}$ & $\begin{array}{c}0.45 \\
(0.15)\end{array}$ & 0.63 & $\begin{array}{c}0.47 \\
(0.15)\end{array}$ & $\begin{array}{c}0.48 \\
(0.13)\end{array}$ & $\begin{array}{c}0.49 \\
(0.13)\end{array}$ & 0.31 & $\begin{array}{c}0.41 \\
(0.13)\end{array}$ & $\begin{array}{c}0.37 \\
(0.17)\end{array}$ & $\begin{array}{c}0.36 \\
(0.15)\end{array}$ & 0.61 \\
\hline Step time (s) & $\begin{array}{c}0.85 \\
(0.49)\end{array}$ & $\begin{array}{c}0.80 \\
(0.37)\end{array}$ & $\begin{array}{c}0.79 \\
(0.38)\end{array}$ & 0.97 & $\begin{array}{c}0.67 \\
(0.18)\end{array}$ & $\begin{array}{c}0.65 \\
(0.09)\end{array}$ & $\begin{array}{c}0.62 \\
(0.06)\end{array}$ & 0.90 & $\begin{array}{c}1.19 \\
(0.69)\end{array}$ & $\begin{array}{c}1.08 \\
(0.51)\end{array}$ & $\begin{array}{c}1.12 \\
(0.51)\end{array}$ & 0.56 \\
\hline Step width (m) & $\begin{array}{c}0.19 \\
(0.07)\end{array}$ & $\begin{array}{c}0.18 \\
(0.07)\end{array}$ & $\begin{array}{c}0.19 \\
(0.07)\end{array}$ & 0.82 & $\begin{array}{c}0.20 \\
(0.07)\end{array}$ & $\begin{array}{c}0.20 \\
(0.08)\end{array}$ & $\begin{array}{c}0.20 \\
(0.08)\end{array}$ & 0.94 & $\begin{array}{l}0.15 \\
(0.04)\end{array}$ & $\begin{array}{l}0.15 \\
(0.03)\end{array}$ & $\begin{array}{l}0.16 \\
(0.05)\end{array}$ & 0.69 \\
\hline Stride length (m) & $\begin{array}{l}0.87 \\
(0.31)\end{array}$ & $\begin{array}{c}0.88 \\
(0.30)\end{array}$ & $\begin{array}{c}0.89 \\
(0.31)\end{array}$ & 0.56 & $\begin{array}{c}0.95 \\
(0.29)\end{array}$ & $\begin{array}{c}0.95 \\
(0.26)\end{array}$ & $\begin{array}{c}0.99 \\
(0.27)\end{array}$ & 0.33 & $\begin{array}{c}0.73 \\
(0.33)\end{array}$ & $\begin{array}{c}0.73 \\
(0.33)\end{array}$ & $\begin{array}{c}0.72 \\
(0.32)\end{array}$ & 0.46 \\
\hline Stride time (s) & $\begin{array}{l}1.69 \\
(1.0)\end{array}$ & $\begin{array}{c}1.60 \\
(0.76)\end{array}$ & $\begin{array}{c}1.59 \\
(0.77)\end{array}$ & 0.96 & $\begin{array}{c}1.33 \\
(0.36)\end{array}$ & $\begin{array}{c}1.29 \\
(0.19)\end{array}$ & $\begin{array}{c}1.24 \\
(0.12)\end{array}$ & 0.65 & $\begin{array}{c}2.39 \\
(1.42)\end{array}$ & $\begin{array}{c}2.20 \\
(1.06)\end{array}$ & $\begin{array}{l}2.25 \\
(1.04)\end{array}$ & 0.64 \\
\hline Walking speed (m/s) & $\begin{array}{c}0.65 \\
(0.34)\end{array}$ & $\begin{array}{c}0.66 \\
(0.32)\end{array}$ & $\begin{array}{c}0.68 \\
(0.33)\end{array}$ & 0.65 & $\begin{array}{c}0.77 \\
(0.31)\end{array}$ & $\begin{array}{c}0.77 \\
(0.27)\end{array}$ & $\begin{array}{c}0.82 \\
(0.26)\end{array}$ & 0.63 & $\begin{array}{c}0.44 \\
(0.29)\end{array}$ & $\begin{array}{c}0.47 \\
(0.33)\end{array}$ & $\begin{array}{c}0.43 \\
(0.29)\end{array}$ & 0.46 \\
\hline
\end{tabular}

t1, before therapy; 2 , right after therapy; 33,6 weeks after therapy.
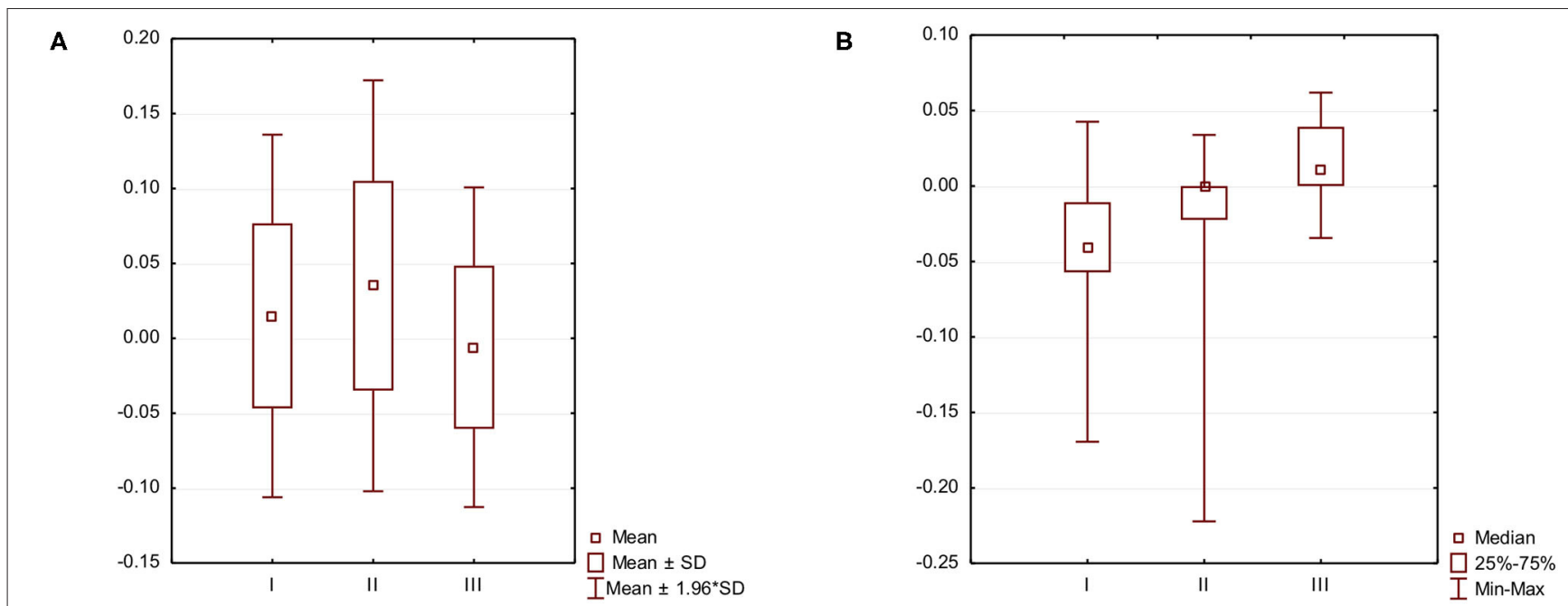

FIGURE 1 | Gait symmetry: (A) spatiotemporal parameter symmetry for Group NAS + AS; (B) walking speed symmetry for Group AS.

\section{DISCUSSION}

The primary aim of the study was to assess the effects of RAT on selected objective gait parameters in hypertonic children with
CP. We did not find any significant effect of RAT on majority of gait parameters except for the improvement in walking speed symmetry (Group AS: $\mathrm{t} 1$ vs. $\mathrm{t} 3, p=0.02$ ) and improvement in double support symmetry (Group AS: $\mathrm{t} 2$ vs. $\mathrm{t} 3, p=0.03$ ). 
TABLE 2 | Changes in gait symmetry between tests.

\begin{tabular}{|c|c|c|c|c|c|c|c|c|c|c|c|c|}
\hline \multirow[t]{2}{*}{ Parameter } & \multicolumn{4}{|c|}{ NAS + AS $(n=26)$} & \multicolumn{4}{|c|}{ NAS $(n=17)$} & \multicolumn{4}{|c|}{ AS $(n=9)$} \\
\hline & $\begin{array}{c}\text { Mean } \\
\text { (SD) } \\
\text { t1 }\end{array}$ & $\begin{array}{c}\text { Mean } \\
\text { (SD) } \\
\text { t2 }\end{array}$ & $\begin{array}{c}\text { Mean } \\
\text { (SD) } \\
\text { t3 }\end{array}$ & $p$ & $\begin{array}{c}\text { Mean } \\
\text { (SD) } \\
\text { t1 }\end{array}$ & $\begin{array}{c}\text { Mean } \\
\text { (SD) } \\
\text { t2 }\end{array}$ & $\begin{array}{c}\text { Mean } \\
\text { (SD) } \\
\text { t3 }\end{array}$ & $p$ & $\begin{array}{c}\text { Mean } \\
\text { (SD) } \\
\text { t1 }\end{array}$ & $\begin{array}{c}\text { Mean } \\
\text { (SD) } \\
\text { t2 }\end{array}$ & $\begin{array}{c}\text { Mean } \\
\text { (SD) } \\
\text { t3 }\end{array}$ & $p$ \\
\hline Cadence (step/min) & $\begin{array}{c}-0.01 \\
(0.02)\end{array}$ & $\begin{array}{r}-0.01 \\
(0.04)\end{array}$ & $\begin{array}{c}0.00 \\
(0.03)\end{array}$ & 0.81 & $\begin{array}{l}-0.01 \\
(0.02)\end{array}$ & $\begin{array}{l}-0.01 \\
(0.03)\end{array}$ & $\begin{array}{c}0.00 \\
(0.02)\end{array}$ & 0.69 & $\begin{array}{l}-0.01 \\
(0.03)\end{array}$ & $\begin{array}{r}-0.01 \\
(0.05)\end{array}$ & $\begin{array}{c}0.00 \\
(0.03)\end{array}$ & 0.26 \\
\hline Double support (s) & $\begin{array}{c}0.01 \\
(0.06)\end{array}$ & $\begin{array}{c}0.04 \\
(0.07)\end{array}$ & $\begin{array}{l}-0.01 \\
(0.05)\end{array}$ & $0.03^{\star}$ & $\begin{array}{c}0.00 \\
(0.05)\end{array}$ & $\begin{array}{c}0.04 \\
(0.08)\end{array}$ & $\begin{array}{c}0.00 \\
(0.06)\end{array}$ & 0.05 & $\begin{array}{c}0.04 \\
(0.08)\end{array}$ & $\begin{array}{c}0.03 \\
(0.06)\end{array}$ & $\begin{array}{l}-0.02 \\
(0.04)\end{array}$ & 0.16 \\
\hline Foot off (\%) & $\begin{array}{c}-0.02 \\
(0.18)\end{array}$ & $\begin{array}{c}0.00 \\
(0.17)\end{array}$ & $\begin{array}{c}0.00 \\
(0.12)\end{array}$ & 0.54 & $\begin{array}{c}-0.03 \\
(0.09)\end{array}$ & $\begin{array}{c}-0.02 \\
(0.08)\end{array}$ & $\begin{array}{c}-0.02 \\
(0.07)\end{array}$ & 0.94 & $\begin{array}{c}0.01 \\
(0.28)\end{array}$ & $\begin{array}{c}0.03 \\
(0.27)\end{array}$ & $\begin{array}{c}0.05 \\
(0.17)\end{array}$ & 0.24 \\
\hline Opposite foot contact (\%) & $\begin{array}{c}-0.02 \\
(0.22)\end{array}$ & $\begin{array}{r}-0.02 \\
(0.19)\end{array}$ & $\begin{array}{c}-0.03 \\
(0.17)\end{array}$ & 0.45 & $\begin{array}{c}-0.06 \\
(0.15)\end{array}$ & $\begin{array}{c}-0.05 \\
(0.15)\end{array}$ & $\begin{array}{c}-0.06 \\
(0.10)\end{array}$ & 0.66 & $\begin{array}{c}0.06 \\
(0.30)\end{array}$ & $\begin{array}{c}0.04 \\
(0.27)\end{array}$ & $\begin{array}{c}0.03 \\
(0.25)\end{array}$ & 0.61 \\
\hline Opposite foot off (\%) & $\begin{array}{c}-0.02 \\
(0.33)\end{array}$ & $\begin{array}{c}-0.03 \\
(0.31)\end{array}$ & $\begin{array}{c}-0.13 \\
(0.31)\end{array}$ & 0.81 & $\begin{array}{c}-0.07 \\
(0.27)\end{array}$ & $\begin{array}{c}-0.04 \\
(0.13)\end{array}$ & $\begin{array}{c}-0.13 \\
(0.21)\end{array}$ & 0.33 & $\begin{array}{c}0.08 \\
(0.42)\end{array}$ & $\begin{array}{c}-0.02 \\
(0.35)\end{array}$ & $\begin{array}{l}-0.13 \\
(0.47)\end{array}$ & 0.27 \\
\hline Single support (s) & $\begin{array}{c}-0.04 \\
(0.29)\end{array}$ & $\begin{array}{r}-0.02 \\
(0.26)\end{array}$ & $\begin{array}{c}0.01 \\
(0.23)\end{array}$ & 0.36 & $\begin{array}{c}-0.07 \\
(0.18)\end{array}$ & $\begin{array}{c}-0.05 \\
(0.31)\end{array}$ & $\begin{array}{c}-0.03 \\
(0.16)\end{array}$ & 0.33 & $\begin{array}{c}0.02 \\
(0.43)\end{array}$ & $\begin{array}{c}0.04 \\
(0.42)\end{array}$ & $\begin{array}{c}0.10 \\
(0.32)\end{array}$ & 0.64 \\
\hline Step length (m) & $\begin{array}{c}-0.06 \\
(0.31)\end{array}$ & $\begin{array}{l}-0.07 \\
(0.48)\end{array}$ & $\begin{array}{c}0.07 \\
(0.37)\end{array}$ & 0.23 & $\begin{array}{c}-0.06 \\
(0.30)\end{array}$ & $\begin{array}{c}-0.05 \\
(0.13)\end{array}$ & $\begin{array}{c}-0.02 \\
(0.23)\end{array}$ & 0.90 & $\begin{array}{l}-0.06 \\
(0.37)\end{array}$ & $\begin{array}{l}-0.12 \\
(0.75)\end{array}$ & $\begin{array}{c}0.24 \\
(0.52)\end{array}$ & 0.14 \\
\hline Step time (s) & $\begin{array}{c}0.02 \\
(0.22)\end{array}$ & $\begin{array}{c}0.03 \\
(0.19)\end{array}$ & $\begin{array}{c}0.03 \\
(0.16)\end{array}$ & 0.34 & $\begin{array}{c}0.07 \\
(0.16)\end{array}$ & $\begin{array}{c}0.06 \\
(0.29)\end{array}$ & $\begin{array}{c}0.06 \\
(0.09)\end{array}$ & 0.91 & $\begin{array}{l}-0.06 \\
(0.30)\end{array}$ & $\begin{array}{c}-0.03 \\
(0.27)\end{array}$ & $\begin{array}{l}-0.02 \\
(0.25)\end{array}$ & 0.68 \\
\hline Step width (m) & $\begin{array}{c}0.02 \\
(0.08)\end{array}$ & $\begin{array}{c}0.01 \\
(0.10)\end{array}$ & $\begin{array}{c}-0.02 \\
(0.13)\end{array}$ & 0.47 & $\begin{array}{c}0.02 \\
(0.09)\end{array}$ & $\begin{array}{c}0.00 \\
(0.14)\end{array}$ & $\begin{array}{c}-0.03 \\
(0.15)\end{array}$ & 0.36 & $\begin{array}{c}0.03 \\
(0.06)\end{array}$ & $\begin{array}{c}0.03 \\
(0.07)\end{array}$ & $\begin{array}{c}0.01 \\
(0.09)\end{array}$ & 0.70 \\
\hline Stride length (m) & $\begin{array}{c}0.00 \\
(0.05)\end{array}$ & $\begin{array}{c}0.01 \\
(0.04)\end{array}$ & $\begin{array}{c}0.01 \\
(0.03)\end{array}$ & 0.16 & $\begin{array}{c}0.01 \\
(0.03)\end{array}$ & $\begin{array}{c}0.01 \\
(0.12)\end{array}$ & $\begin{array}{c}0.00 \\
(0.02)\end{array}$ & 0.32 & $\begin{array}{l}-0.02 \\
(0.06)\end{array}$ & $\begin{array}{c}0.01 \\
(0.05)\end{array}$ & $\begin{array}{c}0.02 \\
(0.04)\end{array}$ & 0.10 \\
\hline Stride time (s) & $\begin{array}{c}0.01 \\
(0.02)\end{array}$ & $\begin{array}{c}0.01 \\
(0.04)\end{array}$ & $\begin{array}{c}0.00 \\
(0.03)\end{array}$ & 0.76 & $\begin{array}{c}0.01 \\
(0.02)\end{array}$ & $\begin{array}{c}0.01 \\
(0.03)\end{array}$ & $\begin{array}{c}0.00 \\
(0.02)\end{array}$ & 0.66 & $\begin{array}{c}0.02 \\
(0.03)\end{array}$ & $\begin{array}{c}0.01 \\
(0.05)\end{array}$ & $\begin{array}{c}0.00 \\
(0.03)\end{array}$ & 0.80 \\
\hline Walking speed (m/s) & $\begin{array}{l}-0.01 \\
(0.05)\end{array}$ & $\begin{array}{l}-0.01 \\
(0.05)\end{array}$ & $\begin{array}{c}0.01 \\
(0.03)\end{array}$ & 0.47 & $\begin{array}{c}0.00 \\
(0.03)\end{array}$ & $\begin{array}{c}0.00 \\
(0.03)\end{array}$ & $\begin{array}{c}0.00 \\
(0.02)\end{array}$ & 0.83 & $\begin{array}{l}-0.04 \\
(0.06)\end{array}$ & $\begin{array}{c}-0.03 \\
(0.07)\end{array}$ & $\begin{array}{c}0.02 \\
(0.03)\end{array}$ & $0.02^{*}$ \\
\hline
\end{tabular}

t1, before therapy; $t 2$, right after therapy; $t 3,6$ weeks after therapy; ${ }^{\star}$ Statistically significant.

At $\mathrm{t} 1$, there was a slight asymmetry to the right side of the body $(\mathrm{SI}=0.01)$. Right after the therapy $(\mathrm{t} 2)$, the asymmetry shifted more toward the right side of the body $(\mathrm{SI}=0.04)$. At $\mathrm{t} 3$, the asymmetry shifted to the opposite direction to the left $(\mathrm{SI}=-0.01)$. The secondary aim of the study was to determine whether changes in selected objective gait parameters are different among children in different ambulatory levels. We did not find differences in changes in selected objective gait parameters between children walking with or without aids.

\section{Therapeutic Effects of RAT}

A significant benefit from the RAT was only noticed in two parameters of walking symmetry. It is not clear if this limited, although statistically significant improvement in symmetry of walking has any impact on the clinical or functional condition of a patient. The possible explanation of these improvements is our training protocols. Except pure gait training, the therapy was focused on load distribution between body sides (gamma dynamographic platforms) and balancing of center of pressure in all directions (alfa stabilometric platform). Patients were trained with virtual reality games to gain better control of the loading of body weight on the left and right foot. Moreover, the exoskeleton force subjects to take more regular steps. It can explain the prominent changes in some parameters of gait symmetry. Surprisingly, we did not notice any improvement in spatiotemporal parameters and pattern of walking.

It is worth emphasizing that despite the fact that changes in GDI were not statistically significant, we still observed improvements in some patients $(\mathrm{t} 2=15.4 \%$; $3=26.9 \%)$. Specific analysis on patients whose gait pattern improved or deteriorated could provide the answer who will possibly benefit from this kind of therapy program the most.

In addition, outcome measures used in this study were highly variable, which was confirmed by non-parametric distribution. The variability was conspicuous especially in Group AS. The explanation of such highly scattering results can be due to individuals with all levels of the ambulation abilities (i.e., GMFCS levels I to IV) were included.

\section{Inconsistency to Previous Studies}

Our findings are in contrary to the majority of previous reports. Previous studies reported significant benefits of RAT for the patient with neurological deficits, including improvement of gait parameters, balance and functional status (changes of gross motor function measure [GMFM] total score and dimensions D \& E scores). Important changes were shown in self-paced gait velocity of walking, step length, cadence, muscles activity, and kinematic data of the knee movement (25-30). In contrary, our data between three visits ( $t 1, t 2$, and $t 3$ ) showed that neither 
TABLE 3 | Number of participants who had changes in GDI.

\begin{tabular}{lcc}
\hline Category & $\begin{array}{c}\text { t2 vs. t1 } \\
\boldsymbol{n}=\mathbf{2 6}\end{array}$ & $\begin{array}{c}\text { t3 vs. t1 } \\
\boldsymbol{n}=\mathbf{2 6}\end{array}$ \\
\hline Improvement & 4 & 7 \\
Deterioration & $(15.4 \%)$ & $(26.9 \%)$ \\
& 6 & 6 \\
No changes & $(23.1 \%)$ & $(23.1 \%)$ \\
& 16 & 13 \\
& $(61.5 \%)$ & $(50 \%)$ \\
\hline
\end{tabular}

right after therapy ( $\mathrm{t} 2)$ nor 6 weeks after the last session ( $\mathrm{t} 3$ ), changes in the kinematics of gait (GDI), symmetry (assessed for all parameters) and spatiotemporal parameters did not occur. Bayón et al. showed that better benefits were achieved by more affected individuals than those on higher functional level (29). According to our results, there were no changes in the most outcome measures at $\mathrm{t} 2$ or $\mathrm{t} 3$ among children in different ambulatory levels, except speed symmetry, which improves only in the more affected group, i.e., the Group AS. Mean value of spatiotemporal parameters, SI or GDI did not change neither in NAS nor in AS which suggested that functional status of subjects and effects of RAT might not be directly related. We believe these disagreements in findings could be explained:

First, the sample size of research group improvement in spatiotemporal parameters was shown in studies based on a relatively small number of participants (26-31). The impact of ambulation level on therapy benefits was shown in group of 4 merely subjects (29). There was only one study with a similar number of patients to our study, where therapy was based on locomotor treadmill. However, no significant changes in spatiotemporal parameters were found in this study either (12). Our study groups consist of 26 subjects with all levels of ambulatory function (GMFCS levels I to IV) which may reflect the therapeutic effect to individuals affected by different severity of CP.

Second, the training and examination protocols are different from study to study. Some researchers showed data collected during the therapy session (improvement in crouch gait, electromyographic activity) and proved that the equipment can change the pattern of gait, but did not show its effectiveness for daily walking ability $(12,31-36)$. The effects of RAT analysis vary from 1 to 20 session which were conducted from five visits over 12 weeks to five times per week. The therapy in our study lasted for 10 weeks and each patient received 30 training sessions, five sessions per week. Moreover, we decided to combine gait and balance training as strongly indissoluble activities. The shortterm effect of the RAT was evaluated twice: right after therapy was completed and 6 weeks later.

Last, the equipment varies from study to study. The effectiveness of different kinds of an exoskeleton, CP walkers, treadmills, and virtual reality training sometimes combined with conventional therapy, were used. In our study, patients received combined therapy of exoskeleton, treadmill, platforms, and virtual reality video game, all within one session. Our intention was to assess pure RAT. In theory, this kind of intervention is supposed to understand needs and requirements of the subject easier than a conventional one. Also, it is easier to adjust the training program to demands of an individual.

\section{Limitations}

One major limitation of this study is the data analysis method used. We have used an average of three trials. For those patients whose gait pattern is very consistent, it is a reasonable method. However, in our research group, we had patients at GMFCS level IV whose stride-to-stride variability is commonly high. Bulea et al. suggested using the variance ratio for kinematic data processing to evaluate the repeatability of results in a more reasonable way in these cases (36).

No matched control group was used in the present study due to the clinical restriction. A full-scale case-control study will help to clarify unanswered questions.

Results of this study do not provide strong evidence to support that RAT can be highly beneficial for individuals with spastic CP in improving gait functions. However, the length of follow-up was only after one training protocol, and only part of the gait parameters was investigated. We did not take the endurance of walking or general gross motor function into consideration. Future research projects on the effect of therapy intensity with similar protocols and other outcome measures are required to clarify the most suitable candidates and the optimal therapy dose for RAT. Moreover, it is also very important to evaluate electromyography, to assess the effect on possible neural structure reorganization.

\section{CONCLUSION}

Our data suggest that RAT based on our protocol does not change spatiotemporal parameters and kinematics of walking except for limited improvement in some aspects of gait symmetry. Moreover, we did not find differences in changes in selected objective gait parameters among children in different ambulatory levels.

\section{DATA AVAILABILITY STATEMENT}

The data that support the findings of this study are available from the corresponding author upon reasonable request. The data are not publicly available due to privacy or ethical restrictions.

\section{ETHICS STATEMENT}

The studies involving human participants were reviewed and approved by Ethical Committee-Poznań University of Medical Sciences, Poland. Written informed consent to participate in this study was provided by the participants' legal guardian.

\section{AUTHOR CONTRIBUTIONS}

FM: contributes to the study concept and design, data collection, data analysis, manuscript drafting, and final manuscript approval. SB and AK: contributes to the data collection, data 
analysis, and final manuscript approval. MJ: contributes to the study design, data analysis, and final manuscript approval. All the authors have read and agreed to the published version of the manuscript.

\section{REFERENCES}

1. Bjornson KF, Belza B, Kartin D, Logsdon R, McLaughlin JF. Ambulatory physical activity performance in youth with cerebral palsy and youth who are developing typically. Phys Ther. (2007) 87:248-57. doi: 10.2522/ptj.20060157

2. Kerr C, McDowell B, McDonough S. The relationship between gross motor function and participation restriction in children with cerebral palsy: an exploratory analysis. Child Care Health Dev. (2007) 33:22-7. doi: 10.1111/j.1365-2214.2006.00634.x

3. Jaspers E, Verhaegen A, Geens F, Van Campenhout A, Desloovere K, Molenaers G. Lower limb functioning and its impact on quality of life in ambulatory children with cerebral palsy. Eur J Paediatr Neurol. (2013) 17:561-7. doi: 10.1016/j.ejpn.2013.04.006

4. Moreau NG, Bodkin AW, Bjornson K, Hobbs A, Soileau M, Lahasky K. Effectiveness of rehabilitation interventions to improve gait speed in children with cerebral palsy: systematic review and meta-analysis. Phys Ther. (2016) 96:1938-54. doi: 10.2522/ptj.20150401

5. Cherng RJ, Liu CF, Lau TW, Hong RB. Effect of treadmill training with body weight support on gait and gross motor function in children with spastic cerebral palsy. Am J Phys Med Rehabil. (2007) 86:548-55. doi: 10.1097/PHM.0b013e31806dc302

6. Provost B, Dieruf K, Burtner PA, Phillips JP, Bernitsky-Beddingfield A, Sullivan KJ, et al. Endurance and gait in children with cerebral palsy after intensive body weight-supported treadmill training. Pediatr Phys Ther. (2007) 19:2-10. doi: 10.1097/01.pep.0000249418.25913.a3

7. Willoughby KL, Dodd KJ, Shields N. A systematic review of the effectiveness of treadmill training for children with cerebral palsy. Disabil Rehabil. (2009) 31:1971-9. doi: 10.3109/09638280902874204

8. Lefmann S, Russo R, Hillier S. The effectiveness of robotic-assisted gait training for paediatric gait disorders: systematic review. J Neuroeng Rehabil. (2017) 14:1. doi: 10.1186/s12984-016-0214-x

9. Booth AT, Buizer AI, Meyns P, Oude Lansink IL, Steenbrink F, van der Krogt MM. The efficacy of functional gait training in children and young adults with cerebral palsy: a systematic review and meta-analysis. Dev Med Child Neurol. (2018) 60:866-83. doi: 10.1111/dmcn.13708

10. Swinnen E, Duerinck S, Baeyens JP, Meeusen R, Kerckhofs E. Effectiveness of robot-assisted gait training in persons with spinal cord injury: a systematic review. J Rehabil Med. (2010) 42:520-6. doi: 10.2340/16501977-0538

11. Schroeder AS, Von Kries R, Riedel C, Homburg M, Auffermann H, Blaschek A, et al. Patient-specific determinants of responsiveness to robot-enhanced treadmill therapy in children and adolescents with cerebral palsy. Dev Med Child Neurol. (2014) 56:1172-9. doi: 10.1111/dmcn.12564

12. Druzbicki M, Rusek W, Snela S, Dudek J, Szczepanik M, Zak E, et al. Functional effects of robotic-assisted locomotor treadmill therapy in children with cerebral palsy. J Rehabil Med. (2013) 45:358-63. doi: 10.2340/16501977-1114

13. Nikityuk IE, Moshonkina TR, Shcherbakova NA, Vissarionov SV, Umnov VV, Rozhdestvenskii VY, et al. Effect of locomotor training and functional electrical stimulation on postural function in children with severe cerebral palsy. Hum Physiol. (2016) 42:262-70. doi: 10.1134/S0362119716030129

14. Brütsch K, Koenig A, Zimmerli L, Mérillat-Koeneke S, Riener R, Jäncke $\mathrm{L}$, et al. Virtual reality for enhancement of robot-assisted gait training in children with neurological gait disorders. J Rehabil Med. (2011) 43:493-9. doi: 10.2340/16501977-0802

15. Meyer-Heim A, Borggraefe I, Ammann-Reiffer C, Berweck S, Sennhauser FH, Colombo G, et al. Feasibility of robotic-assisted locomotor training in children with central gait impairment. Dev Med Child Neurol. (2007) 49:900-6. doi: 10.1111/j.1469-8749.2007.00900.x

16. van Hedel HJA, Meyer-Heim A, Rüsch-Bohtz C. Robot-assisted gait training might be beneficial for more severely affected children with cerebral

\section{FUNDING}

This study was supported by Polish/National Center for Research and Development (NCBR; Grant \# RPWP.01.02.00-30-0118/17).

palsy. Dev Neurorehabil. (2016) 19:410-5. doi: 10.3109/17518423.2015.1 017661

17. Meyer-Heim A, Ammann-Reiffer C, Schmartz A, Schaefer J, Sennhauser $\mathrm{FH}$, Heinen $\mathrm{F}$, et al. Improvement of walking abilities after robotic-assisted locomotion training in children with cerebral palsy. Arch Dis Childhood. (2009) 94:615-20. doi: 10.1136/adc.2008.145458

18. Borggraefe I, Meyer-Heim A, Kumar A, Schaefer JS, Berweck S, Heinen F. Improved gait parameters after robotic-assisted locomotor treadmill therapy in a 6-year-old child with cerebral palsy. Move Disord. (2008) 23:280-3. doi: $10.1002 / \mathrm{mds} .21802$

19. Bayon C, Ramírez O, Velasco M, Serrano JI, Lara SL, MartinezCaballero I, et al. Pilot study of a novel robotic platform for gait rehabilitation in children with cerebral palsy. In: 2016 6th IEEE International Conference on Biomedical Robotics Biomechatronics (BioRob). IEEE (2016). doi: 10.1109/BIOROB.2016.7523739

20. Borggraefe I, Kiwull L, Schaefer JS, Koerte I, Blaschek A, Meyer-Heim A, et al. Sustainability of motor performance after robotic-assisted treadmill therapy in children: an open, non-randomized baseline-treatment study. Eur J Phys Rehabil Med. (2010) 46:125-31. doi: 10.5167/uzh-40098

21. Rajagopal A, Kidziński Ł, McGlaughlin AS, Hicks JL, Delp SL, Schwartz MH. Estimating the effect size of surgery to improve walking in children with cerebral palsy from retrospective observational clinical data. Sci Rep. (2018) 8:1. doi: 10.1038/s41598-018-33962-2

22. Schwartz MH, Rozumalski A, Steele KM. Dynamic motor control is associated with treatment outcomes for children with cerebral palsy. Dev Med Child Neurol. (2016) 58:1139-45. doi: 10.1111/dmcn.13126

23. Robinson RO, Herzog W, Nigg BM. Use of force platform variables to quantify the effects of chiropractic manipulation on gait symmetry. J Manipulative Physiol Ther. (1987) 10:172-6.

24. Schwartz MH, Rozumalski A. The gait deviation index: A new comprehensive index of gait pathology. Gait Posture. (2008) 28:351-7. doi: 10.1016/j.gaitpost.2008.05.001

25. Mutlu A, Krosschell K, Spira DG. Treadmill training with partial body-weight support in children with cerebral palsy: a systematic review. Dev Med Child Neurol. (2009) 51:268-75. doi: 10.1111/j.1469-8749.2008.03221.x

26. Lotfian M, Dadashi F, Rafieenazari Z, Shahroki A, Rasteh M, Molavi M, et al. The effects of anti-gravity treadmill training on gait characteristics in children with cerebral palsy. In: 2019 41st Annual International Conference of the IEEE Engineering in Medicine Biology Society (EMBC). IEEE (2019). doi: 10.1109/EMBC.2019.8856660

27. Ueno $T$, Watanabe $H$, Kawamoto $H$, Shimizu $Y$, Endo A, Shimizu $\mathrm{T}$, et al. Feasibility and safety of Robot Suit HAL treatment for adolescents and adults with cerebral palsy. J Clin Neurosci. (2019) 68:101-4. doi: 10.1016/j.jocn.2019.07.026

28. Smania N, Bonetti P, Gandolfi M, Cosentino A, Waldner A, Hesse S, et al. Improved gait after repetitive locomotor training in children with cerebral palsy. Am J Phys Med Rehabil. (2011) 90:137-49. doi: 10.1097/PHM.0b013e318201741e

29. Bayón C, Martín-Lorenzo T, Moral-Saiz B, Ramírez Ó, Pérez-Somarriba Á, Lerma-Lara S, et al. A robot-based gait training therapy for pediatric population with cerebral palsy: goal setting, proposal and preliminary clinical implementation. J Neuroeng Rehabil. (2018) 15:1-5. doi: 10.1186/s12984-018-0412-9

30. Digiacomo F, Tamburin S, Tebaldi S, Pezzani M, Tagliafierro M, Casale $\mathrm{R}$, et al. Improvement of motor performance in children with cerebral palsy treated with exoskeleton robotic training: A retrospective explorative analysis. Restorative Neurol Neurosci. (2019) 37:239-44. doi: 10.3233/RNN180897

31. Lerner ZF, Damiano DL, Park HS, Gravunder AJ, Bulea TC. A robotic exoskeleton for treatment of crouch gait in children with cerebral palsy: 
Design and initial application. IEEE Trans Neural Syst Rehabil Eng. (2016) 25:650-9. doi: 10.1109/TNSRE.2016.2595501

32. Aycardi LF, Cifuentes CA, Múnera M, Bayón C, Ramírez O, Lerma S, et al. Evaluation of biomechanical gait parameters of patients with Cerebral Palsy at three different levels of gait assistance using the CPWalker. J Neuroeng Rehabil. (2019) 16:1-9. doi: 10.1186/s12984-019-0485-0

33. Bulea TC, Lerner ZF, Gravunder AJ, Damiano DL. Exergaming with a pediatric exoskeleton: facilitating rehabilitation and research in children with cerebral palsy. In: 2017 International Conference on Rehabilitation Robotics (ICORR). IEEE (2017). doi: 10.1109/ICORR.2017.80 09394

34. Lerner ZF, Harvey TA, Lawson JL. A battery-powered ankle exoskeleton improves gait mechanics in a feasibility study of individuals with cerebral palsy. Ann Biomed Eng. (2019) 47:1345-56. doi: 10.1007/s10439-019-0 2237-W

35. Lerner ZF, Damiano DL, Bulea TC. A lower-extremity exoskeleton improves knee extension in children with crouch gait from cerebral palsy. Sci Transl Med. (2017) 9:9145. doi: 10.1126/scitranslmed. aam9145

36. Bulea TC, Lerner ZF, Damiano DL. Repeatability of EMG activity during exoskeleton assisted walking in children with cerebral palsy: implications for real time adaptable control. Conf Proc IEEE Eng Med Biol Soc. (2018) 2018:2801-4. doi: 10.1109/EMBC.2018.8512799

Conflict of Interest: The authors declare that the research was conducted in the absence of any commercial or financial relationships that could be construed as a potential conflict of interest.

Publisher's Note: All claims expressed in this article are solely those of the authors and do not necessarily represent those of their affiliated organizations, or those of the publisher, the editors and the reviewers. Any product that may be evaluated in this article, or claim that may be made by its manufacturer, is not guaranteed or endorsed by the publisher.

Copyright (C) 2021 Manikowska, Brazevic, Krzyżańska and Jóźwiak. This is an openaccess article distributed under the terms of the Creative Commons Attribution License (CC BY). The use, distribution or reproduction in other forums is permitted, provided the original author(s) and the copyright owner(s) are credited and that the original publication in this journal is cited, in accordance with accepted academic practice. No use, distribution or reproduction is permitted which does not comply with these terms. 